\title{
Uma Análise do Uso da Ontologia IMS LD na Construção de Modelos Conceituais para E-learning *
}

\author{
Francisco Hélio de Oliveira ${ }^{1}$, Laís do Nascimento Salvador ${ }^{1,2}$, Renato Novais ${ }^{3}$ \\ ${ }^{1}$ Departamento de Ciência da Computação - Universidade Federal da Bahia (UFBA) \\ ${ }^{2}$ Fraunhofer Project Center for Software and Systems Engineering, Bahia, Brasil \\ ${ }^{3}$ Instituto Federal da Bahia (IFBA) \\ \{franciscohelio, laisns\}@dcc.ufba.br, renato@ifba.edu.br
}

\begin{abstract}
Difficulty in phase requirements elicitation is a problem that affects the modeling software. Errors at this stage give rise to conceptual models that do not properly represent the user's interest. Domain ontologies can be used to minimize this problem. The aim of this study is to show that the use of IMS LD ontology may result in more accurate educational software. To this end, experimental studies where the traditional approach to requirements elicitation was confronted with the approach that uses this ontology were performed. The results were evaluated by statistical analysis. The main result of this study proves to be feasible using the IMS LD ontology as input in conceptual modeling.
\end{abstract}

Resumo. Dificuldades na fase de elicitação de requisitos constituem um problema que afeta a modelagem de software. Erros nesta fase dão origem a modelos conceituais que não representam corretamente o interesse do usuário. Por sua vez, ontologias de domínio podem ser usadas para minimizar esse problema. O objetivo deste estudo é mostrar que o uso da ontologia IMS LD pode resultar em software educacional mais correto. Para tal, foram realizados estudos experimentais em que a abordagem tradicional de elicitação de requisitos foi confrontada com a abordagem que usa esta ontologia. Os resultados foram avaliados através de análises estatísticas. $O$ principal resultado deste estudo demonstra a viabilidade do uso da ontologia IMS LD como subsídio na modelagem conceitual.

\section{Introdução}

Uma das atividades mais complexas do processo de Engenharia de Software é a elicitação de requisitos, na qual analistas interagem com diversas fontes de informação (e.g. stakeholders) para descobrir as principais funções de um sistema e restrições sobre seu funcionamento. Várias dificuldades, tais como inconsistências e conflitos de interesse, devem ser contornadas para que, ao final do processo, a especificação do sistema seja válida, completa e consistente [Borst 1997]. Nesse contexto, a dificuldade de comunicação entre analistas e stakeholders é um problema clássico a ser tratado

\footnotetext{
* Esse projeto foi apoiado pelo Instituto Nacional de Ciência e Tecnologia para Engenharia de Software (INES) com apoio financeiro do CNPq (projeto 5773964/2008-4).
} 
[Guarino 1998]. Esse problema se agrava quando o trabalho está relacionado com domínios mais complexos, tais como padrões de Educação a Distância (EAD).

Ontologias podem ser utilizadas no processo de extração de conhecimento do domínio de interesse, e consequentemente minimizar as dificuldades de elicitação de requisitos. Uma ontologia pode ser definida como uma descrição formal de um domínio do discurso, e destina-se a partilhar conhecimento entre diferentes aplicações. Ela é expressa em uma linguagem que pode ser usada para o raciocínio [Noy 2004]. As ontologias estão assumindo um papel importante como um elemento de origem na criação de modelos de domínio de software [Pinto et al. 2011]. Como exemplo, cita-se a ontologia IMS Learning Desing (IMS LD) [Amorim et al. 2006], criada para a modelagem conceitual de projetos educacionais.

A especificação IMS LD tem como uma de suas características, o desenvolvimento de uma estrutura que apoia a diversidade pedagógica e inovação, bem como promove o intercâmbio e a interoperabilidade de materiais de e-learning. Ela propõe também um padrão para modelar projetos de aprendizagem, que não está relacionado somente com ambiente de e-learning [Silva and Santachè 2009]. Entretanto, autores destacam tanto a dificuldade de construir software educacional [Marczak et al. 2003] quanto em manter ambientes de aprendizagem [Bittencourt et al. 2006].

Por sua vez, Vasilecas et al. (2009) afirmam que o uso de ontologia pode reduzir a complexidade da modelagem conceitual. Entretanto, não foi encontrado na literatura nenhum estudo experimental que avalie essa afirmação. Este trabalho tem a intenção de preencher esta lacuna. O estudo conduzido avalia a efetividade do uso de ontologia IMS LD na modelagem de um sistema educacional, comparando-o ao uso tradicional isolado do documento de requisitos.

O artigo está estruturado da seguinte forma: a Seção 2 apresenta a fundamentação teórica necessária para compreensão dos conceitos utilizados. A Seção 3 descreve a metodologia utilizada no trabalho. A Seção 4 descreve a avaliação experimental. A Seção 5 discute os resultados obtidos. A Seção 6 discute alguns trabalhos relacionados. Por fim, a Seção 7 apresenta as considerações finais.

\section{Fundamentação Teórica}

Modelos conceituais são usados para explicitar características e comportamentos de um software e estão intimamente ligados à qualidade da implementação dos requisitos desejados pelo usuário. A fase de modelagem é essencial no processo de desenvolvimento de software em qualquer domínio. Franciosi et al. (2002) destaca essa importância na construção de ambientes de aprendizagem. Nesta seção é apresentada a ontologia IMS LD desenvolvida em OWL [OWL 2004] e também é discutido um paralelo entre ontologias e modelos conceituais.

\subsection{Ontologia IMS LD}

Ontologia é uma especificação formal de uma conceptualização compartilhada. Ela geralmente é construída por especialistas no domínio de interesse, e neste processo de construção enfatiza-se o fato de que deve haver um consenso sobre os elementos que são especificados [Borst 1997]. Mais especificamente, uma ontologia de domínio descreve o vocabulário relacionado a um domínio genérico, especializando os termos da ontologia 
de alto nível onde são definidos os conceitos gerais que podem estar associados a mais de um domínio [Guarino 1998].

A ontologia de domínio IMS LD representa a semântica da especificação IMS Learning Design. Esta especificação utiliza-se da descrição do processo educacional em Unidades de Aprendizagem com o objetivo de elaborar um fluxo de aprendizagem. Este fluxo se desenvolve a partir da realização de atividades, apoiadas pelo uso de materiais digitais e ferramentas de interação (Objetos de Aprendizagem e Serviços). Tais ferramentas são definidas a partir de objetivos de aprendizagem em um determinado planejamento didático. De acordo com [Koper and Olivier 2004], uma Unidade de Aprendizagem pode ser vista como um nome genérico para um curso, um workshop, uma lição, etc. Ela pode ser instanciada e reutilizada várias vezes por diferentes pessoas e configurações em um ambiente on-line.

Neste contexto, a ontologia IMS LD foi desenvolvida com o propósito de se obter uma descrição formal mais precisa facilitando o desenvolvimento de aplicações que implementam a especificação associada. Esta ontologia possui os seguintes elementos: classes, propriedades e indivíduos. Ela se diferencia pelo uso de restrições que são representadas de uma forma declarativa, formal e independente de linguagem. Para isso, são utilizados axiomas declarados em lógica.

\subsection{Ontologias e Modelos Conceituais}

Com o aumento da complexidade dos softwares educacionais, da diversidade de tecnologias adotadas e do grande número de pessoas envolvidas, tornou-se inadequado projetar um programa educacional sem utilizar-se de um processo bem definido para orientar o seu desenvolvimento [Marczak et al. 2003]. Nesta linha o presente estudo mostra o uso de uma ontologia como apoio no processo de desenvolvimento de software para a área educacional. Esta é uma abordagem promissora, uma vez que ontologias buscam fornecer uma conceituação explícita sobre seus elementos, ajudando as pessoas a compreenderem melhor a área de conhecimento [Gava and Menezes 2003].

No desenvolvimento de software, uma das tarefas iniciais - após o levantamento de requisitos - é a definição do modelo conceitual [Wazlawick 2010]. Modelo conceitual aqui pode ser entendido como uma abstração da realidade de acordo com uma determinada conceptualização [Guizzardi 2005]. E conceptualização como o conjunto de conceitos utilizados para articular abstrações de estado de coisas em um determinado domínio. Ainda, de acordo com [Guizzardi 2007] a especificação de um modelo conceitual subjacente a uma linguagem é a descrição do que as primitivas dessa linguagem são capazes de representar em termos de fenômeno do mundo real. Observase assim uma semelhança e interseção entre as definições de modelo conceitual e de ontologia de domínio. A especificação destes dois conceitos é usualmente feita em UML [UML 2011], no caso de modelos conceituais, e em OWL [OWL 2004] no caso de ontologias de domínio. Uma das iniciativas de "casar" estes dois universos é o padrão ODM - Ontology Definition Metamodel - [ODM 2009] da Object Management Group (OMG).

A Tabela I apresenta uma correspondência entre conceitos OWL e elementos do diagrama de classes, base dos modelos conceituais descritos em UML. 
TABELA I - ELEMENTOS COMUNS ENTRE UML E OWL [VASILECAS ET AL. 2009][ODM 2009][HORRIDGE 2011]

\begin{tabular}{|l|l|l|}
\hline \multicolumn{1}{|c|}{ Elemento OWL } & Elemento UML & \multicolumn{1}{c|}{ Descrição } \\
\hline $\begin{array}{l}\text { Classe } \\
\text { owl: class }\end{array}$ & Classe & $\begin{array}{l}\text { Conjuntos que agrupam indivíduos ou } \\
\text { instâncias/objetos do mesmo tipo. }\end{array}$ \\
\hline $\begin{array}{l}\text { Indivíduo } \\
\text { class } \\
\text { rdf:ID=individu } \\
\text { o }\end{array}$ & Instância/objeto & $\begin{array}{l}\text { Indivíduos representam objetos (instâncias) } \\
\text { no domínio de interesse, isto é, objetos de } \\
\text { uma classe. }\end{array}$ \\
\hline $\begin{array}{l}\text { Propriedade de Objeto } \\
\text { owl: } \text { objectPrope } \\
\text { rty }\end{array}$ & Associação & $\begin{array}{l}\text { Propriedades de objetos são relações } \\
\text { binárias entre dois indivíduos que podem } \\
\text { ser convertidas (com restrições) em } \\
\text { associações entre classes em UML }\end{array}$ \\
\hline $\begin{array}{l}\text { Taxonomia } \\
\text { rdfs: subclassof }\end{array}$ & $\begin{array}{l}\text { Herança operador de taxonomia subclassof } \\
\text { relaciona uma classe mais específica com } \\
\text { uma classe mais geral. Esta relação pode } \\
\text { ser convertida no conceito de herança em } \\
\text { UML onde uma classe (subclasse) herda } \\
\text { dados e comportamento de outra classe } \\
\text { (superclasse). }\end{array}$ \\
\hline
\end{tabular}

Os conceitos apresentados possuem basicamente a mesma semântica em cada uma das linguagens, como é o caso dos conceitos de classe, instância/indivíduo e herança. A única observação é para o mapeamento de propriedade de objeto OWL em associação UML. Este mapeamento funciona apenas se a cardinalidade tanto do domínio, quanto do escopo (range), da propriedade for unitária (1 classe no domínio e 1 classe no escopo). Vale ressaltar que as relações de composição e agregação de UML são de difícil visualização na ontologia, pois OWL não apresenta construtores específicos para expressar estes conceitos. Entretanto, a construção owl:objectProperty aliada ao uso de restrições (allValuesFrom e someValuesFrom) também pode ser usada para ajudar na elicitação das relações de composição e agregação ${ }^{1}$.

\section{Metodologia}

Para determinar o quanto uma ontologia de domínio pode ajudar na criação de modelos conceituais na área de e-learning foi planejado e executado um experimento controlado. Este estudo cumpriu as seguintes etapas: i) planejamento de um experimento controlado; ii) avaliação dos procedimentos do experimento através de estudos pilotos; iii) aplicação do experimento; iv) aplicação de estatística apropriada para análise dos dados. v) análise e discussão dos resultados. Uma descrição do experimento aplicado é mostrada na Seção 4.

\footnotetext{
${ }^{1}$ Relações todo-parte em OWL: http://www.w3.org/2001/sw/BestPratics/OEP/SimplePartWhole/
} 


\section{Estudo Experimental}

O objetivo deste estudo foi investigar evidências de que a abordagem baseada no uso de ontologias de domínio nas fases iniciais do desenvolvimento de software facilita a construção de sistemas de informação na área de e-learning. Para tal, foi conduzido um experimento controlado no qual participantes realizaram atividades de modelagem em um sistema EAD.

Considerando a abordagem baseada em ontologias para construção de sistemas de informação, planeja-se caracterizar sua diferença com relação à abordagem tradicional (sem o uso de ontologias), com respeito à corretude e eficiência. Um modelo (diagrama de classe) de referência testado e validado foi utilizado como oráculo no experimento. Para a corretude foram avaliados e contados classes, associações, agregações, heranças e composições pertencentes ao modelo de referência que determinado indivíduo conseguiu identificar. A eficiência foi medida através do registro do tempo total (em minutos) de realização das atividades por parte dos participantes.

A identificação dos objetivos deste estudo foi feita segundo a abordagem Goal Question Metric (GQM) [Basili and Rombach 1988]. O objetivo do estudo foi avaliar o uso da ontologia IMS LD na modelagem de sistema, com o propósito de caracterizar o impacto da adoção de ontologias na modelagem conceitual, tendo com foco de qualidade a corretude da modelagem e tempo de desenvolvimento do diagrama de classe, do ponto de vista dos pesquisadores deste estudo, no contexto da análise de desenvolvimento de software por alunos de pós-graduação, e profissionais (desenvolvedores de software e analistas de sistemas).

Este experimento contou com 29 participantes. Eles foram selecionados entre alunos do curso de mestrado em Ciência da Computação da UFBA, alunos de doutorado em Ciência da Computação da UFBA e profissionais de duas empresas de Vitória da Conquista - BA. Os participantes foram separados em dois Grupos (GC, controle, sem o uso da ontologia; GE, experimental, com uso da ontologia). Cada participante foi alocado a um grupo de acordo com sua experiência e de forma a manter os dois grupos equilibrados.

Os dois grupos que compõem o experimento receberam materiais diferentes. $\mathrm{O}$ GC recebeu apenas um texto referente ao documento de requisitos. Esse documento, escrito em português, apresentava todas as classes e relações pertencentes à parte do domínio do problema (área de e-learning). O GE recebeu, além do texto, a ontologia IMS LD (taxonomia). Essa ontologia, escrita em inglês, continha as classes do domínio. O GE recebeu ainda uma tabela em português com a descrição de todas as objectProperty da ontologia. O material continha 19 classes: act, academic, activity, structured-activity, environment, evaluation, learn-activity, object-activity, objectivelearning, learner, method, play, prerequisite, professional, role, role-part, service, staff, support-activity. Entre as classes existem as seguintes relações: 6 associações, 6 heranças, 8 agregações, 3 composições. Independente dos documentos utilizados, cada participante teve que produzir um diagrama de classe.

O experimento continha 5 tarefas, a saber: Tarefa 1 (T1), encontrar as classes corretamente como descritas no modelo de referência; Tarefa 2 (T2), encontrar todas as relações de herança corretamente como descritas no modelo de referência; Tarefa 3 
(T3), encontrar todas as relações de associação corretamente como descritas no modelo de referência; Tarefa 4 (T4), encontrar todas as relações de composição corretamente como descritas no modelo de referência; Tarefa 5 (T5), encontrar todas as relações de agregação corretamente como descritas no modelo de referência.

Duas hipóteses foram elaboradas para este experimento: H1: a abordagem baseada em ontologia produz artefato mais correto que a abordagem sem ontologia; $\mathrm{H} 2$ : a abordagem baseada em ontologia diminui o tempo gasto na construção do modelo conceitual. As hipóteses nulas são a negação de cada uma das hipótese H1 e H2.

Para determinar a corretude dos artefatos produzidos pelos participantes a seguinte métrica foi adotada: para cada elemento encontrado corretamente em uma tarefa foi contado o valor $\mathrm{x}$ (onde $\mathrm{x}=1$ /número de elementos corretos do oráculo) e para cada erro -x. Como exemplo pode ser citada a relação de agregação que aparece 8 vezes no oráculo. Assim, para cada agregação encontrada corretamente foi contado $1 / 8=$ 0,125 pontos. Foi considerada correta a tarefa que obteve o valor 1 , parcialmente correta aquela que obteve um valor no intervalo ]0,1[, e incorreta para os casos de valor menor ou igual a 0 . Os critérios foram definidos de acordo com [Novais et al. 2012].

Este experimento contém um fator (técnica ou abordagem) e dois tratamentos (com ontologia e sem ontologia). A taxonomia da ontologia IMS LD foi mostrada através de uma figura e as propriedades de objeto através de uma tabela contendo o nome da propriedade, o domínio (domain) e o escopo (range) utilizando a codificação Manchester [OWL 2 2012]. Todos os participantes foram treinados na linguagem UML. Os participantes do GE foram treinados com conceitos de ontologia. Os participantes preencheram um questionário de perfil onde classificaram seu conhecimento em relação à linguagem, isto é, o conhecimento formal construído a partir de algum curso na área de informática. Os participantes ficaram assim distribuídos: 2 experientes, 10 com conhecimento moderado, 4 com pouco conhecimento e 3 nenhum. Para compor o perfil também foi perguntado aos participantes sobre sua experiência efetiva no uso da linguagem UML através de tempo de trabalho em desenvolvimento de software. Com relação à experiência: 4 trabalharam 3 anos ou mais; 3 entre 2 e 3 anos; 7 entre 1 e 2 anos; 5 entre 6 meses e 1 ano; 1 menos de 6 meses e 9 nunca trabalharam na área.

Os procedimentos para execução do experimento foram os seguintes: i) os participantes assinaram a lista de presença com um identificador único; ii) foram distribuídos os artefatos aos participantes, de acordo com o grupo a que pertenciam, a folha de resposta e o Termo de Consentimento; iii) o termo de consentimento foi lido, assinado e recolhido; iv) foi feita uma leitura dos artefatos; v) foi proposto como objetivo: obtenção do diagrama de classe que representa o modelo conceitual do Learning Design com classes e relações; vi) cada participante desenhou seu diagrama de classe na sua folha de resposta, indicando o tempo inicial e final de realização do experimento; vii) após a entrega da folha de resposta o participante preencheu o questionário de feedback; viii) cada diagrama de classe obtido pelos participantes foi comparado com o do modelo de referência, para verificação da corretude e eficiência.

\section{Análise e Resultados}

Após a execução do experimento, cinco participantes foram caracterizados com outliers. Desta forma ficou-se com 24 participantes, 12 para cada grupo. A Figura 1 apresenta o 
número de respostas por categoria (errado, parcialmente errado, certo), por tipo de tarefa (e.g. classe, relação) e por grupo (GC e GE).

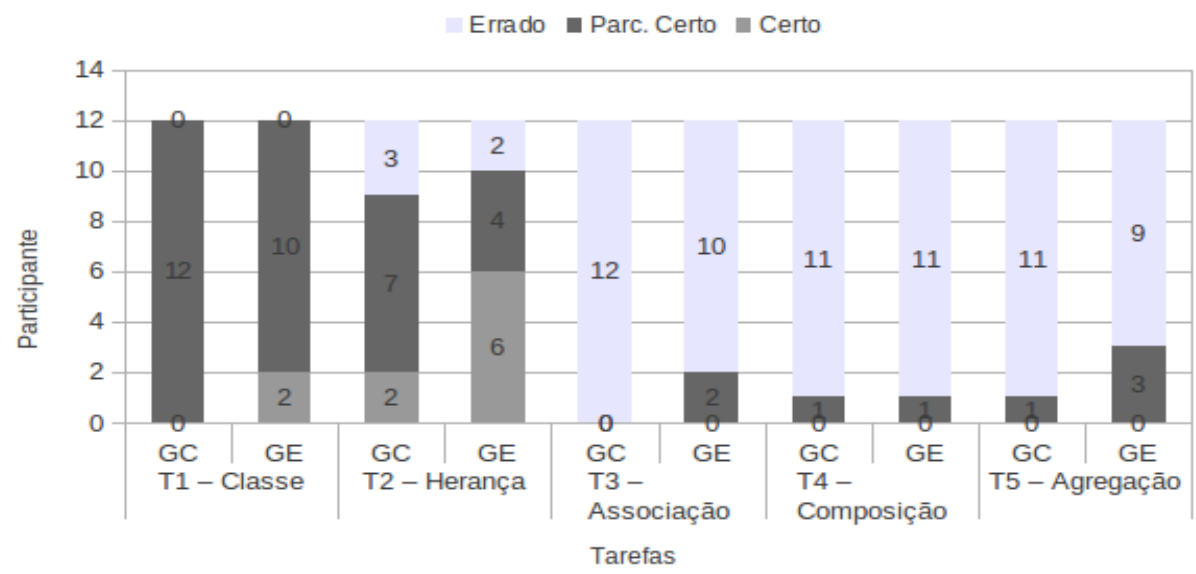

Figura 1 - Tarefas por grupo

Os resultados mostram que há uma predominância de acertos e acertos parciais para as tarefas T1 e T2. As tarefas T3, T4 e T5 mostram uma superioridade no número de erros com $100 \%$ na tarefa T3 para o grupo de controle. Pode-se observar que a abordagem que usa ontologia pode ser superior em relação à abordagem tradicional exceto na tarefa T4 - Composição. Após realizar o experimento, foram obtidos os valores de corretude e tempo para cada um dos grupos. Para ambos, os testes estatísticos foram utilizados considerando o nível de confiança de 95\% (alpha=0,05). $\mathrm{O}$ valor final da corretude de um participante é calculado através da soma da corretude conseguida em cada tarefa. O tempo total é calculado através da diferença entre o tempo final e o tempo inicial.

TABELA II - ESTATÍSTICA DESCRITIVA DOS RESULTADOS DO EXPERIMENTO

\begin{tabular}{|c|c|c|c|c|c|c|c|c|c|}
\hline & Grupo & Média & Diferença & Min & Max & $\begin{array}{l}\text { Desv. } \\
\text { Padrão }\end{array}$ & $\begin{array}{l}\text { S-W } \\
\text { p-value }\end{array}$ & Levene & $\begin{array}{l}\text { t-test } \\
p \text { - } \\
\text { value }\end{array}$ \\
\hline \multirow{2}{*}{$\begin{array}{c}\text { Tempo } \\
\text { (minutos) }\end{array}$} & GC & 48,33 & & 38 & 64 & 6,89 & & & \\
\hline & GE & 50,92 & $+5,31 \%$ & 33 & 82 & 13,49 & & & \\
\hline \multirow{2}{*}{$\begin{array}{l}\text { Corretude } \\
\text { (pontos) }\end{array}$} & GC & 8,6 & & 0 & 19 & 6,64 & 0,9222 & 0,6838 & 0,229 \\
\hline & GE & 12,4 & $+44,1 \%$ & 1 & 26 & 7,90 & 0,3646 & & \\
\hline
\end{tabular}

O uso da ontologia mostrou uma média de acertos maior que a abordagem sem ontologia. O grupo que utilizou a ontologia se mostrou superior nas tarefas T1, T2, T4 e T5. Considerando todas as tarefas o grupo experimental foi superior em $44,1 \% \mathrm{em}$ média ao grupo de controle como mostra a Tabela II. Entretanto, não foi possível obter significância estatística através dos testes realizados. Uma das causas para isso pode ser o pequeno número de participantes no experimento.

Como uma classe extraída do texto pode ser autenticada na ontologia sua presença no diagrama pode ficar mais clara. A extração da herança a partir da taxonomia 
da ontologia facilita sua identificação. E por fim, as propriedades de objeto da ontologia que relaciona duas classes, uma no domínio e outra no escopo, facilitam a conclusão de que essas duas classes tem uma relação de associação, composição ou agregação. Essas características tendem a ajudar na construção do diagrama de classe.

Todos os participantes iniciaram o experimento ao mesmo tempo e ao término das tarefas o tempo final foi marcado. Desse modo, foi obtida a quantidade de tempo, em minutos, que cada um deles gastou. A Tabela II mostra a média de tempo que os grupos gastaram. Pode ser visto que o grupo experimental gastou mais tempo que o grupo de controle $(+5.31 \%)$, contrariando a hipótese de que o grupo experimental gastaria menos tempo para a realização das tarefas. A amostra de tempo não apresentou vantagem em termos de eficiência em favor da abordagem com ontologia. Alguns dos participantes do GE reportaram que, apesar da ontologia ter ajudado no processo como um todo, no início tiveram dificuldades com o entendimento da ontologia, uma vez que a mesma estava em inglês.

\section{Trabalhos Relacionados}

Diversos trabalhos da literatura discutem o uso ontologias no desenvolvimento de software. Marczak et al. (2003) discutem a modelagem de software para ambientes de aprendizagem. Os autores mostram a modelagem e planejamento de um ambiente utilizando a tecnologia de sistemas Web, a fim de constituir um framework para dar suporte aos alunos e professores em um ambiente de aprendizagem. Pinto et al. (2011) também discutem o uso da ontologia como ferramenta de apoio à modelagem. Eles apresentam a ferramenta MDAOnto que usa a ontologia IMS LD para a criação de software para e-learning. A ferramenta recebe como entrada a ontologia. $\mathrm{O}$ usuário pode interagir para remover conceitos sem relevância para o sistema a ser desenvolvido. Em seguida essa ontologia passa por uma transformação OWL2UML obtendo-se um diagrama de classe.

Modelos baseados em classe UML e ontologias OWL constituem em abordagens de modelagem com diferentes pontos fortes e fracos. Em [Parreiras and Staab 2010], os autores propõem uma utilização integrada de ambas as abordagens de modelagem em um framework (TwoUse). O framework tem uma abordagem que permite construir modelos UML com expressividade semântica da OWL DL.

Pode ser visto que existem estudos relacionando UML e ontologias no sentido de otimizar a criação de modelos conceituais nas fases iniciais de desenvolvimento de software. O diferencial deste trabalho está no uso integrado da ontologia com práticas tradicionais de desenvolvimento de software. Não havendo necessidade de implementação de ferramentas de software para sua utilização. E na ausência de qualquer plataforma de desenvolvimento uma vez que é utilizada a linguagem UML para produção de modelos conceituais para ambientes de aprendizagem.

\section{Conclusões}

O uso de ontologias provavelmente ajuda na modelagem conceitual de sistemas de informação para área de e-learning, pois pode evitar que o analista considere na sua modelagem classes e relações que não são de interesse dentro do contexto. 
As relações de herança são de fácil visualização e identificação e, portanto, demandam pouco trabalho do analista. As relações de composição e agregação têm um grau maior de dificuldade de serem encontradas, pois não estão explicitamente escritas na ontologia OWL. Assim, tanto associação quanto composição e agregação devem ser identificadas de forma cuidadosa através das propriedades de objeto. Porém, o documento de requisitos pode ser utilizado para diminuir a incerteza de qual relacionamento se trata. Dessa forma, o experimento mostrou indícios de que o uso de ontologia ajuda na modelagem conceitual de sistemas de informação. Especificamente, concluiu-se que possivelmente a ontologia IMS LD pode ajudar a modelagem conceitual para e-learning culminando em software mais correto.

Este trabalho também contribui na busca por uma abordagem experimental na área de desenvolvimento dirigido por ontologias. Como trabalhos futuros, pretende-se: (i) estender o experimento considerando mais participantes e variando as tarefas a serem desenvolvidas; (ii) aprofundar o estudo e avaliação da extração de relações de composição/agregação em ontologias de domínio escritas em OWL.

\section{Referências}

Amorim, R., Lama, M., Sánchez, E., Riera, A., and Vila, X. A. (2006) "Learning Design Ontology based on the IMS Specification", In: Journal of Educational Technology Society, pages 38-57.

Basili, V. and Rombach, H.D. (1988) "The TAME Project: Towards ImprovementOriented Software Environments", IEEE Transactions on Software Engineering, vol. 14, pages 758-773.

Bittencourt, I., Nunes, C., Costa, E., Tadeu, M and Bezerra, C. (2006). 'Um Sistema de Autoria para Construção de Ambientes Interativos de Aprendizagem Baseado em Agentes”, Anais do XVII SBIE - Simpósio Brasileiro de Informática na Educação, UNB/UCB, pages 378-387.

Borst, W. N. (1997) "Construction of Engineering Ontologies for Knowledge Sharing and Reuse." Thesis Enschede - No. 97-14.

Franciosi, B. R. T., Medeiros, M. F. de, Vargas, R. F., Pernigotti, J. M., Marques, J. C. and Colla, A. L. (2002) "Experiência de Modelagem de Ambientes de Aprendizagem da PUCRS VIRTUAL", Anais do XII SBIE - Simpósio Brasileiro de Informática na Educação, Unisinos, pages 178-183.

Gava, T. B. S. and Menezes, C. S. de (2003). "Uma ontologia de domínio para a aprendizagem cooperativa”. Anais do XIV SBIE - Simpósio Brasileiro de Informática na Educação, NCE - IM/UFRJ, pages 336-345.

Guarino, N. (1998) "Formal Ontology and Information Systems.", In: Proceedings of FOIS'98, Trento, Italy. Amsterdam, IOS Press, pages 3-15.

Guizzardi, G. (2007) "On Ontology, Ontologies, Conceptualizations, Modeling Languages, and (Meta)Models", Proceedings of the 2007 conference on Databases and Information Systems IV: Selected Papers from the Seventh International Baltic Conference DB\&IS'2006. pages 18-39. 
Guizzardi, G. (2005) “Ontological Foundations For Structural Conceptual Models", CTIT PhD.-thesis series, No. 05-74 ISSN 1381-3617; No. Centre for Telematics and Information Technology, University of Twente.

Horridge, M. (2011) “A Practical Guide To Building OWL Ontologies Using Protégé 4 and CO-ODE Tools ", University of Manchester.

IMS Learning Design Information Model: Version 1.0 Final Specification (2003). Disponível em:http://www.imsglobal.org/learningdesign/ldv1p0/imsld_infovlp0.html., acesso em: junho de 2014.

Koper, R. and Olivier, B. (2004) "Representing the Learning Design of Units of Learning”, Educational Technology \& Society, vol. 7, pages 97-111.

Marczak, S., Girafa, L., Almeida, G., Blois and Marcelo B. (2003) "Modelando um ambiente de aprendizagem na Web: a importância da formalização do processo de desenvolvimento", XIV Simpósio Brasileiro de Informática na Educação - NCE IM/UFRJ, pages 535-544.

Noy, N. F. (2004) "Semantic Integration: a Survey of Ontology Based Approaches", In: SIGMOD Record, ACM Press, 33, pages 65-70.

ODM - Ontology Definition Metamodel (2009). OMG (Object Modeling Group), disponível em: http://www.omg.org/spec/ODM/1.0/, acesso em: Junho de 2014.

OWL -Web Ontology Language Guide. Disponível em: http://www.w3.org/TR/owlguide/, acesso em: junho de 2014.

OWL 2 (Web Ontology Language) - Manchester Syntax ( 2012 ), disponível em: http:// www.w3.org / TR / ow12- manchester-syntax /, acesso em: Julho de 2014.

Novais, R., Nunes, C., Cirilo, E., Dantas, F., Garcia, A. and Mendonça, M. (2012) "On the Proactive and Interactive Visualization for Feature Evolution Comprehension: An Industrial Investigation", In: Proceeding of the 34th International Conference on Software Engineering, pages 1044-1053.

Parreiras, F. S. and Staab S. (2010) "Using Ontologies with UML Class-based Modeling: The TwoUse Approach”, In: Data \& Knowledge Engineering. Vol. 69, pages 11941207.

Pinto, G. P., Salvador, L. N. and Amorim, R. (2011) "A Model Driven Solution Based on Ontology in the Implementation of Units of Learning Authoring Tools", IEEE Multidisciplinary Engineering Education Magazine, v.6, pages 31-49.

Silva, L. A. M. da and Santanchè, A. (2009) "Introdução ao Learning Design", Anais do XX SBIE - Simpósio Brasileiro de Informática na Educação, Florianópolis - SC.

UML - Unified Modeling Language. Infrastructure. Version 2.4.1 (2011), disponível em: http://www.omg.org/spec/UML/2.4.1/Infrastructure/PDF/, acesso em: julho de 2014.

Vasilecas, O., Kalibatiene, D. and Guizzardi, G. (2009) “Towards a Formal Method For The Transformation of Ontology Axioms to Application Domain Rules", Information Technology and Control Vol. 38, No.4

Wazlawick, R. S.(2010) Análise e Projeto de Sistemas Orientado a Objetos. Elsevier. 